\title{
Recovery of corneal irregular astigmatism, ocular higher-order aberrations, and contrast sensitivity after discontinuation of overnight orthokeratology
}

T Hiraoka, C Okamoto, Y Ishii, F Okamoto and T Oshika

Br. J. Ophthalmol. 2009;93;203-208; originally published online 19 Nov 2008; doi:10.1136/bjo.2007.136655

Updated information and services can be found at:

http://bjo.bmj.com/cgi/content/full/93/2/203

These include:

References This article cites 27 articles, 5 of which can be accessed free at: http://bjo.bmj.com/cgi/content/full/93/2/203\#BIBL

Rapid responses You can respond to this article at: http://bjo.bmj.com/cgi/eletter-submit/93/2/203

Email alerting Receive free email alerts when new articles cite this article - sign up in the box at service the top right corner of the article

Notes

To order reprints of this article go to:

http://journals.bmj.com/cgi/reprintform

To subscribe to British Journal of Ophthalmology go to:

http://journals.bmj.com/subscriptions/ 


\title{
Recovery of corneal irregular astigmatism, ocular higher-order aberrations, and contrast sensitivity after discontinuation of overnight orthokeratology
}

\author{
T Hiraoka, C Okamoto, Y Ishii, F Okamoto, T Oshika
}

Department of Ophthalmology, Institute of Clinical Medicine, University of Tsukuba, Ibaraki, Japan

\section{Correspondence to:}

Dr T Hiraoka, Department of Ophthalmology, Institute of Clinical Medicine, University of Tsukuba, 1-1-1, Tennoudai, Tsukuba, Ibaraki, 305-8575 Japan;

thiraoka@md.tsukuba.ac.jp

Accepted 21 October 2008 Published Online First

19 November 2008

\begin{abstract}
Aims: To examine prospectively the recovery of various parameters after discontinuation of overnight orthokeratology.

Methods: Seventeen subjects undergoing orthokeratology for 12 months were examined. Refraction, corneal topography, wavefront aberrometry, a visual acuity test and a contrast sensitivity test were performed at baseline, 12 months after commencement of the procedure, and 1 week and 1 month after discontinuation of the treatment. Asymmetry and higher-order irregularity components were calculated using a Fourier analysis of the corneal topography data. Contrast sensitivity was assessed at four spatial frequencies, and the area under the log contrast sensitivity function (AULCSF) was calculated.
\end{abstract}

Results: Orthokeratology significantly reduced manifest refraction ( $p<0.0001$, Dunnett test) and significantly improved uncorrected visual acuity (UCVA) at 12 months after commencement of the procedure $(p<0.0001)$. Asymmetry and higher-order irregularity components increased significantly $(p<0.0001, p=0.0032$, respectively), and third- and fourth-order aberrations also increased significantly $(p<0.0001)$. The treatment resulted in significant decreases in AULCSF $(p=0.0004)$. After discontinuing lens wear, all parameters, such as refraction, UCVA, asymmetry, higher-order irregularity, third-order aberration, fourth-order aberration and AULCSF, returned to the baseline level at 1 week. Conclusion: This study confirmed that the effect of orthokeratology is completely reversible in light of optical quality of the eye and quality of vision as well as refraction and visual acuity.

Overnight orthokeratology is a non-surgical procedure to achieve transient reduction in refractive error and improvement of unaided vision especially in low to moderate myopic patients through a programmed application of specially designed rigid contact lenses which are worn during sleep. This procedure can provide patients with useful vision during waking hours without depending on external corrective devices, such as spectacles and daily wear contact lenses. A number of studies have demonstrated the efficacy of this procedure, ${ }^{1-3}$ and orthokeratology has been widely known as a new alternative option to correct myopia. ${ }^{4}$ One of the proposed advantages of overnight orthokeratology is that its effect is impermanent, unlike corneal refractive surgery. Several short-term studies have reported that the influence of orthokeratology on refraction and visual acuity diminishes once lens wear is discontinued. ${ }^{5-8}$ However, no studies have confirmed complete recovery of refraction and visual acuity after discontinuing the procedure. The main reason may be that the recovery data are difficult to collect, because most successful cases in overnight orthokeratology are not willing to give up lens wear once they are adapted to the procedure, ${ }^{7}$ or patients, even if they quit the treatment, want to start wearing other corrective devices, such as daily-wear contact lenses, immediately after the discontinuation.

In general, contact lenses, regardless of the kinds or wearing modalities, can affect corneal shape and physiology. ${ }^{9-13}$ Ruiz-Montenegro ${ }^{13}$ found that contact lens-induced corneal topographic abnormalities were observed in a significant proportion of patients, and these alterations were more common and severe in rigid contact lens wearers than in soft contact lens wearers. Although such changes are considered to be reversible in most cases, several cases with irreversible changes have been reported. ${ }^{9-12}$ In addition, some reports have shown a possible relationship between contact lens wear and the development of keratoconus. ${ }^{14-16}$ In orthokeratology, the cornea is intentionally moulded by a positive pressure induced by reverse geometry lenses with a very flat base curve. Hence, some practitioners remain skeptical regarding the overall reversibility after discontinuation of the procedure. $^{\text {? }}$

Recently, several studies have demonstrated that overnight orthokeratology causes declines in optical quality of the eye, such as increases in corneal irregular astigmatism ${ }^{17}$ and higher-order wavefront aberrations (HOAs) of the cornea ${ }^{18}$ and the eye. ${ }^{19-21}$ There have been reports that this procedure reduces low-contrast visual acuity. ${ }^{21}$ Furthermore, it has been demonstrated that contrast sensitivity function decreases in parallel with the increases in HOAs following overnight orthokeratology. ${ }^{20}$ The recovery of these parameters after ceasing the treatment, however, has not been studied. Given the growing popularity of orthokeratology, ${ }^{4}$ it is crucial to know whether such declines completely recover after discontinuation of the procedure. Therefore, we conducted the current prospective study to investigate recovery of various clinical parameters including corneal irregular astigmatism, HOAs and contrast sensitivity after the use of reverse geometry contact lenses for overnight orthokeratology for 1 year.

\section{SUBJECTS AND METHODS}

A prospective study was conducted in 46 eyes of 23 subjects. They were consecutively recruited based 
on the following inclusion criteria; manifest spherical equivalent refraction between -4.00 and -1.00 dioptres (D), refractive astigmatism up to $1.00 \mathrm{D}$, best spectacle-corrected visual acuity (BSCVA) of 20/20 or better, mean keratometry reading between 40.00 and $46.25 \mathrm{D}$, no previous experience with orthokeratology, and age between 20 and 37 years. Subjects with clinical evidence of ocular or corneal pathology including keratoconus and dry eye, with a history of ocular disease or previous ocular surgery or with systemic diseases such as diabetes mellitus were excluded from the study. Subjects were instructed to stop wearing their contact lenses for at least 3 weeks prior to the baseline examination. Each subject had a comprehensive baseline examination including manifest refraction, keratometry, corneal topography, wavefront aberrometry, high-contrast visual acuity testing, contrast sensitivity testing and slit-lamp evaluation. All subjects participated in this study with the hope of being free from or less dependent on optical aids during waking hours by improving unaided visual acuity. No subject wished to qualify for occupations requiring specific unaided visual acuity.

Overnight orthokeratology was carried out using four-zone reverse geometry lenses manufactured from BOSTON XO material (Polymer Technology, Wilmington, Massachusetts) with a nominal Dk of $100 \times 10^{-11}\left(\mathrm{~cm}^{2} / \mathrm{s}\right)(\mathrm{ml} \mathrm{O} 2 / \mathrm{ml} \cdot \mathrm{mm} \mathrm{Hg})$, following the fitting guidelines recommended by the manufacturer. After confirmation of a proper trial lens fit, final lens parameters were determined, and a custom lens was then ordered for each eye. After lens dispensing, subjects started to wear their lenses on an overnight-wear basis in a consecutive manner, and thereafter continued the treatment for 12 months. They were instructed to wear their contact lenses every night for at least $7 \mathrm{~h}$. The lens design was modified in case of poor topographical changes or insufficient improvement of uncorrected visual acuity throughout the study period.

Twelve months after starting orthokeratology treatment, they were asked to cease the lens wear. To evaluate the recovery of clinical parameters such as refraction, visual acuity, corneal irregular astigmatism, HOAs and contrast sensitivity, these data were again collected 1 week and 1 month after discontinuation of the treatment. During this period, they were requested to use eyeglasses, and not to wear any contact lenses. At every visit, subjects were examined between 09:00 and 11:00 to minimise the influence of diurnal variation. Stillitano et $a l^{23}$ reported that wavefront aberration and visual acuity were relatively stable during daytime in patients who had undergone overnight orthokeratology for 6 months. The research adhered to the tenets of the Declaration of Helsinki, and was approved by the institutional review board of Tsukuba University Hospital. Written informed consent was obtained from all subjects after the nature and possible consequences of the study had been explained.

Corneal topography was performed with TMS-4 (Tomey, Aichi, Japan), and measurements were repeated at least three times for each eye to obtain a well-focused and properly aligned image of the eye. The dioptric power of all measurement points was downloaded as an ASCII file. Using Fourier harmonic analysis, ${ }^{24}$ the sequence of power data on a ring $\mathrm{i}, \mathrm{Fi}(\sigma)$, was transformed into trigonometric components of the following form: $\operatorname{Fi}(\sigma)=\mathrm{a}_{0}+\mathrm{c}_{1} \cos \left(\sigma-\alpha_{1}\right)+\mathrm{c}_{2} \cos 2\left(\sigma-\alpha_{2}\right)+\mathrm{c}_{3} \cos 3\left(\sigma-\alpha_{3}\right)+$ $\cdots+c_{n} \operatorname{cosn}\left(\sigma-\alpha_{n}\right)$, where $2 \times c_{1}$ represents the asymmetry (tilt or decentration) component, $2 \times c_{2}$ the regular astigmatism component, and $c_{3 \cdot \cdot n}$ the higher-order irregularity component. Among these, $2 \times c_{1}$ (asymmetry) and $c_{3 \cdot n}$ (higher-order irregularity) denote corneal irregular astigmatism in a broad sense, because these components cannot be corrected by a spherocylindrical lens. ${ }^{24}$ Those indices were calculated on rings 1 to 9 , which correspond to the central $3 \mathrm{~mm}$ zone.

Ocular wavefront aberrometry for a $4 \mathrm{~mm}$ pupil was performed with a Hartmann-Shack wavefront analyser (KR9000 PW, Topcon, Tokyo) through a natural pupil without the use of dilating drugs. Detailed explanations of this system can be found elsewhere. ${ }^{25}$ From the obtained data, HOAs were calculated by the normalised Zernike polynomials and expressed as the root mean square (RMS; in micrometres). The RMS of the third-order Zernike coefficients was used to represent comalike aberration, and the RMS of the fourth-order Zernike coefficients was used to denote spherical-like aberrations. For each eye, measurements were repeated at least four times, and three best-focused images were selected. The averaged data of the three selected images were used for subsequent analyses.

Contrast sensitivity under photopic conditions was determined using the CSV-1000E chart (Vector Vision, Greenville, Ohio). The test was performed monocularly with best spectacle correction in the undilated state at $2.5 \mathrm{~m}$. Four spatial frequencies, 3, 6, 12 and 18 cycles per degree (cpd), are present, and each spatial frequency has eight different levels of contrast. The contrast level of the last correct response was recorded as the contrast threshold in logarithmic values for each frequency. From these data, the area under the log contrast sensitivity function (AULCSF) was calculated according to the method of Applegate et al. ${ }^{26}$ This can represent the contrast sensitivity data as a single quantity used to characterise the overall contrast sensitivity function.

For each clinical parameter, the obtained data at baseline, 12 months after commencement of orthokeratology, and 1 week and 1 month after discontinuation of orthokeratology were analysed using repeated-measures analysis of variance (ANOVA) to assess the time course of changes. If significant differences were observed, a Dunnett post-hoc test was performed. Differences were considered statistically significant at $p<0.05$. All statistical analyses were performed using SPSS version 11.0 J software. So that data collected on both eyes could be used appropriately, eye (right or left) was included as a factor in each repeated-measures ANOVA to account for the covariance between eyes. If no significant interaction with the eye factor was found, the averaged data for both eyes were used for the following analyses.

\section{RESULTS}

During the study period, six subjects dropped out of the study. The reasons for discontinuation included poor improvement in uncorrected visual acuity (UCVA) (two subjects), incidence of monocular diplopia (2), poor adaptation owing to lens discomfort (1) and inability to keep follow-up appointments (1). Hence, the scheduled study was successfully carried out in 17 subjects out of 23 subjects who enrolled originally. They were nine males and eight females, and their mean age was 23.9 (SD 3.5) years. Clinical data of 34 eyes from 17 subjects who completed 1-year follow-up were collected and used for the analyses. Because no significant interaction between eyes and time was present in each repeated-measures ANOVA performed ( $p=0.6803$ for manifest refraction, $p=0.3837$ for UCVA, $p=0.9061$ for BSCVA, $p=0.7812$ for regular astigmatism, $p=0.6401$ for asymmetry component, $p=0.7828$ for the higher-order irregularity component, $p=0.9459$ for third-order RMS, $p=0.8858$ for fourth-order RMS and $p=0.8500$ for AULCSF), the data of right and left eyes from a single subject 
were averaged, and the averaged data $(n=17)$ were then used for the following analyses.

Overnight orthokeratology significantly reduced manifest spherical equivalent refraction from $-2.17(0.80) \mathrm{D}$ at baseline to $-0.17(0.30) \mathrm{D}$ at 12 months after commencement of the procedure ( $\mathrm{p}<0.0001$, Dunnett test), and significantly improved UCVA from $0.72(0.29)$ to $-0.06(0.12) \log \mathrm{MAR}(\mathrm{p}<0.0001)$. BSCVA did not change significantly, with $-0.10(0.06)$ before and -0.10 (0.07) $\log$ MAR 12 months after the procedure $(p=0.9226)$. As for corneal astigmatism derived from a Fourier analysis of the topography data, regular astigmatism did not change significantly, with $0.45(0.29) \mathrm{D}$ before treatment and $0.52(0.30) \mathrm{D}$ after treatment for 12 months $(\mathrm{p}=0.1311)$. Asymmetry component significantly increased from $0.23(0.07) \mathrm{D}$ before to $0.73(0.39) \mathrm{D}$ at 12 months after the procedure $(p<0.0001)$, and higher-order irregularity also increased significantly from $0.10(0.02) \mathrm{D}$ to $0.13(0.05) \mathrm{D}$ $(p=0.0032)$. Regarding ocular wavefront aberrations, the treatment resulted in significant increases in third-order RMS for a $4 \mathrm{~mm}$ pupil from $0.074(0.028) \mu \mathrm{m}$ at baseline to 0.247 $(0.120) \mu \mathrm{m}$ after using orthokeratology lenses for 12 months $(\mathrm{p}<0.0001)$ and fourth-order RMS for a $4 \mathrm{~mm}$ pupil from 0.037 $(0.017) \mu \mathrm{m}$ to $0.129(0.052) \mu \mathrm{m}(\mathrm{p}<0.0001)$. AULCSF decreased significantly from 1.447 (0.104) before to 1.316 (0.179) 12 months after the treatment $(p=0.0004)$ (table 1$)$.

The clinical parameters were monitored at 1 week and 1 month after discontinuation of overnight orthokeratology. Each time course of changes is shown in figs $1-5$. Because all parameters showed significant fluctuations during the study period $(p=0.0021 \sim p<0.0001$, repeated-measures ANOVA), a Dunnett post-hoc test was performed to assess the complete recovery to the baseline value after discontinuation of the treatment. All parameters showed rapid recovery after ceasing orthokeratology. Spherical equivalent refraction at 1 week after discontinuation $(-1.93(0.82) \mathrm{D})$ was not significantly different from the baseline value $(-2.17(0.80) \mathrm{D}, \mathrm{p}=0.1182$, Dunnett test). It further recovered to $-2.07(0.86) \mathrm{D}$ at 1 month after discontinuing lens wear with no significant difference from the baseline $(p=0.9291)$ (fig 1). Similarly, UCVA at 1 week after discontinuation (0.64 (0.33) logMAR) was not significantly different from the baseline value $(0.72(0.29) \log M A R, p=0.1825)$, and it further recovered to 0.70 (0.30) $\log$ MAR at 1 month after discontinuing lens wear $(p=0.9931)$ (fig 2). Other parameters completely recovered with no significant difference from the baseline at 1 week after discontinuation ( $p=0.9337 \sim 0.9999$ ), such as asymmetry component, higher-order irregularity component (fig 3), third-order RMS, fourth-order RMS (fig 4) and AULCSF (fig 5). These parameters remained stable on the 1-month examinations (figs 3-5).

\section{DISCUSSION}

As shown in the results, the spherical equivalent refraction was significantly reduced, and UCVA was significantly improved by orthokeratology. However, the treatment led to significant increases in corneal irregular astigmatism and HOAs. Moreover, contrast sensitivity was significantly decreased by the treatment. All these parameters, however, returned to the baseline level 1 week after discontinuation of the procedure. In particular, the indices that represent corneal irregular astigmatism, HOAs and contrast sensitivity function showed complete recovery to the pretreatment level within 1 week after cessation of contact lens wear, and thereafter maintained the recovered level until 1 month. Spherical equivalent refractive errors and UCVA also returned to the baseline level at 1 week after the discontinuation, but these parameters showed a slight trend of

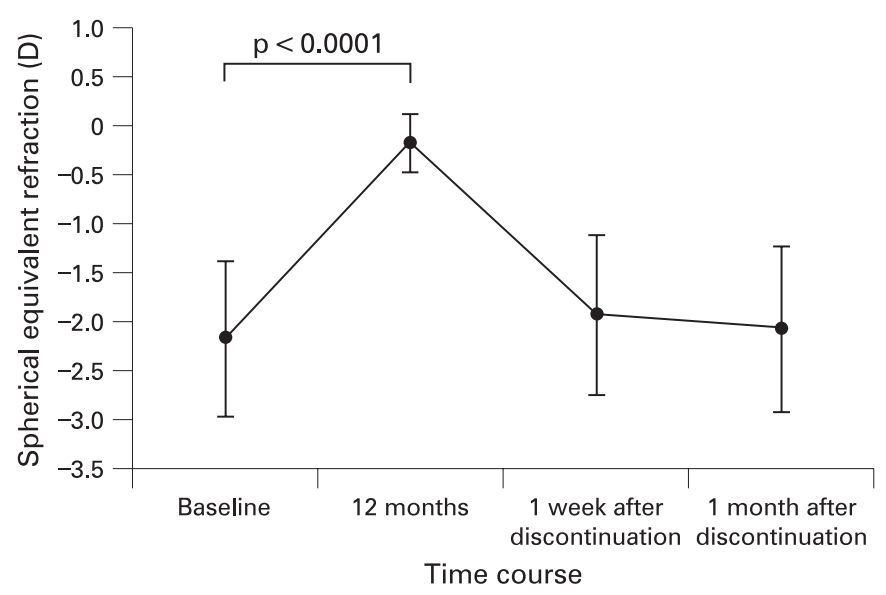

Figure 1 Time course of changes in manifest spherical equivalent refraction after commencement and discontinuation of overnight orthokeratology. Spherical equivalent refraction decreased significantly 12 months after commencement of the procedure $(p<0.0001$, Dunnett test). After discontinuation of the procedure, it returned rapidly, and there was no significant difference between values at baseline and at 1 week after the discontinuation $(p=0.1182)$. One month after the discontinuation, it further recovered to the pretreatment level with no significant difference from the baseline value $(p=0.9291)$. $D=$ dioptre. Error bars represent the SD.

Table 1 Subjects' clinical data at baseline and 12 months after commencement of overnight orthokeratology

\begin{tabular}{lcc}
\hline & Baseline & 12 months after treatment \\
\cline { 2 - 3 } & Mean (SD), range & Mean (SD), range \\
\hline Spherical equivalent refraction (D) & $-2.17(0.80),-3.88$ to -1.06 & $-0.17(0.30),-1.06$ to $0.00^{*}$ \\
UCVA (logMAR) & $0.72(0.29), 0.22$ to 1.15 & $-0.06(0.12),-0.18$ to $0.15^{*}$ \\
BSCVA (logMAR) & $-0.10(0.06),-0.18$ to 0.00 & $-0.10(0.07),-0.18$ to 0.02 \\
Corneal regular astigmatism (D) & $0.45(0.29), 0.08$ to 1.16 & $0.52(0.30), 0.18$ to 1.17 \\
Corneal irregular astigmatism (D) & & \\
$\quad$ Asymmetry & $0.23(0.07), 0.14$ to 0.42 & $0.73(0.39), 0.12$ to $1.42^{*}$ \\
$\quad$ Higher-order irregularity & $0.10(0.02), 0.08$ to 0.14 & $0.13(0.05), 0.09$ to $0.24 *$ \\
Third-order RMS for a $4 \mathrm{~mm}$ pupil $(\mu \mathrm{m})$ & $0.074(0.028), 0.034$ to 0.120 & $0.247(0.120), 0.103$ to $0.491^{*}$ \\
Fourth-order RMS for a $4 \mathrm{~mm}$ pupil $(\mu \mathrm{m})$ & $0.037(0.017), 0.014$ to 0.092 & $0.129(0.052), 0.057$ to $0.233^{*}$ \\
AULCSF & $1.447(0.104), 1.191$ to 1.604 & $1.316(0.179), 0.957$ to $1.598^{*}$ \\
\hline
\end{tabular}

${ }^{*} p<0.0001, \dagger p<0.001, \$ p<0.005$ (Dunnett test): significant differences between baseline and post-treatment values. AULCSF, area under the log contrast sensitivity function; BSCVA, best spectacle-corrected visual acuity; D, dioptre; logMAR, logarithm of the minimum angle of resolution; RMS, root mean square; UCVA, uncorrected visual acuity. 


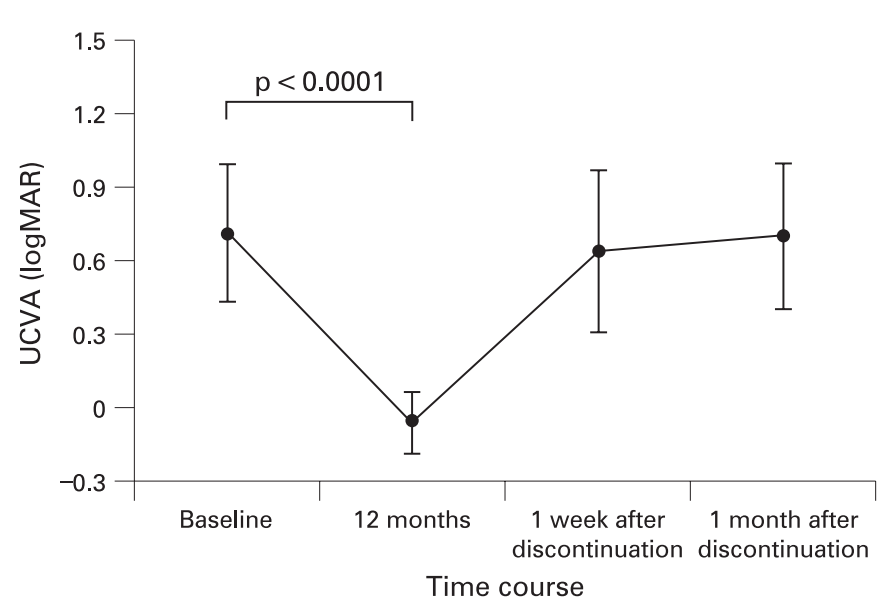

Figure 2 Time course of changes in uncorrected visual acuity (UCVA) after commencement and discontinuation of overnight orthokeratology. UCVA significantly improved at 12 months after commencement of the procedure $(p<0.0001$, Dunnett test). It returned rapidly after discontinuation of the procedure, and significant difference is not noted between values at baseline and at 1 week after the discontinuation $(p=0.1825)$. At 1 month after the discontinuation, it further recovered to the baseline level with no significant difference from the baseline value $(p=0.9931)$. LogMAR = logarithm of the minimum angle of resolution. Error bars represent the SD.

further recovery 1 month after the discontinuation. These findings may indicate that the recovery of refraction and UCVA 1 week after the discontinuation is not enough, although there was no statistically significant difference between the values at baseline and 1 week after the discontinuation. A large sample would have been necessary to determine whether there really was a small further recovery of refraction and UCVA at 1 month after the discontinuation. Further studies should be conducted to confirm this point.

Previous studies revealed that recovery of refractive error toward the baseline after discontinuation of overnight orthokeratology was very rapid over the first $24-72 \mathrm{~h},{ }^{6}$ but complete recovery might require more than 2 weeks. ${ }^{7}$ Soni et al ${ }^{7}$ examined refractive and corneal recovery after the use of reverse-geometry contact lenses for overnight orthokeratology for 1 month, and found that spherical equivalent power and monocular uncorrected visual acuity did not recover fully until 2 weeks after discontinuing lens wear, while central corneal thickness recovered completely after just one night of no lens wear, and central corneal curvature recovered fully in 1 week. From these findings, they speculated that modification of the refractive index of epithelium or other corneal layers might have a greater influence on the refractive error and UCVA than corneal curvature. Also, in our study, the recovery of refractive error and UCVA was somewhat slower than that of other parameters.

As for the reason why corneal irregular astigmatism, HOAs and contrast sensitivity recovered more quickly than refractive error and UCVA, we have no clear explanation at present. However, the corneal surface contour is considered to recover rapidly after discontinuation of orthokeratology as the early recovery of corneal thickness and curvature was confirmed in the previous study, ${ }^{7}$ and thus corneal irregular astigmatism, which reflects subtle changes in corneal surface shape, may have recovered quickly. Regarding ocular HOAs, the changes after orthokeratology are mainly attributed to those in corneal HOAs, because there are few or small changes in internal aberrations due to intraocular optical structures and posterior corneal curvature. ${ }^{1427}$ Besides, corneal HOAs and irregular astigmatism are quite similar parameters representing corneal irregularity which cannot be corrected by spherocylindrical lenses. Therefore, ocular HOAs would have shown a rapid recovery in parallel with corneal irregular astigmatism in our study. As for contrast sensitivity, it is reported to be significantly correlated with ocular HOAs after orthokeratology. ${ }^{20}$ This relationship may have resulted in a similar recovery of contrast sensitivity within 1 week.

There is one limitation in our study. The current study was performed only for adults. Currently, there is no available information on recovery of clinical parameters after
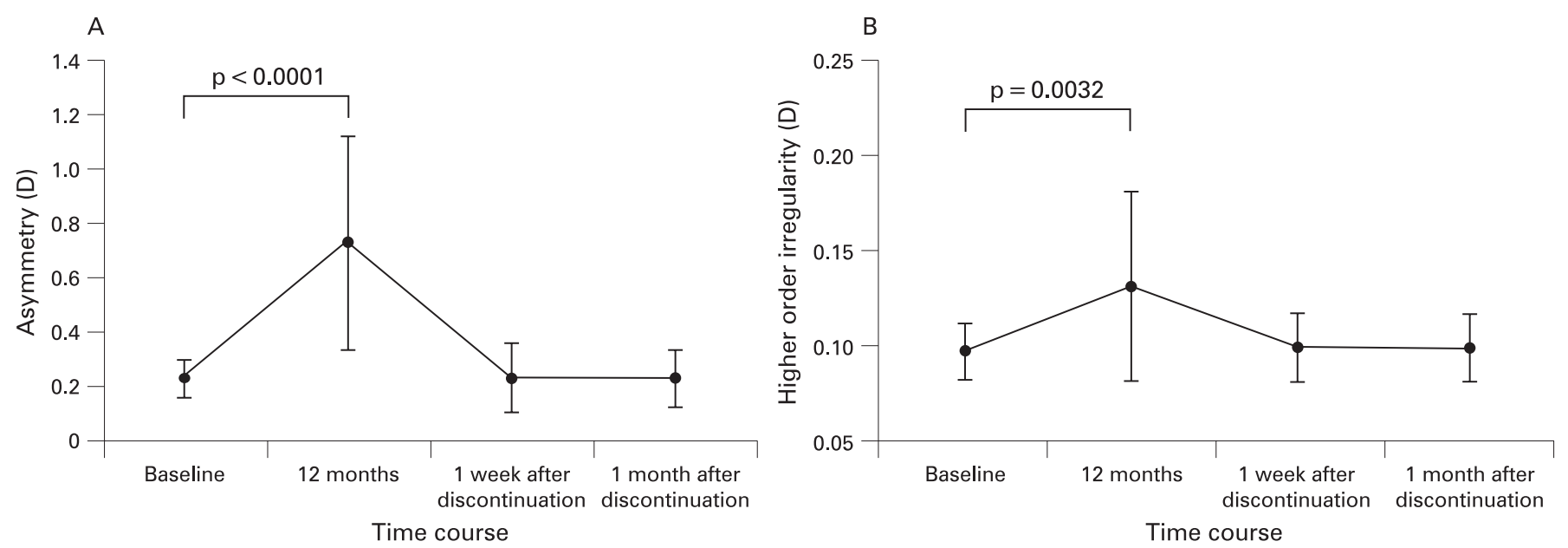

Figure 3 Time course of changes in corneal irregular astigmatism after commencement and discontinuation of overnight orthokeratology. (A) Asymmetry component significantly increased 12 months after commencement of the procedure $(p<0.0001$, Dunnett test). One week after discontinuation of the procedure, it returned fully to the pretreatment level with no significant difference from the baseline value $(p=0.9999)$, and thereafter maintained the recovered level until 1 month without any significant difference from the pretreatment value $(p=0.9999)$. $D=$ dioptre. Error bars represent the SD. (B) Higher order irregularity component significantly increased 12 months after commencement of the procedure $(p=0.0032$, Dunnett test). At 1 week after discontinuation of the procedure, it completely recovered without significant difference from the baseline value $(p=0.9988)$. One month after the discontinuation, it remained at the recovered level with no significant difference from the pretreatment value $(p=0.9976) . D=$ dioptre. Error bars represent the SD. 
A

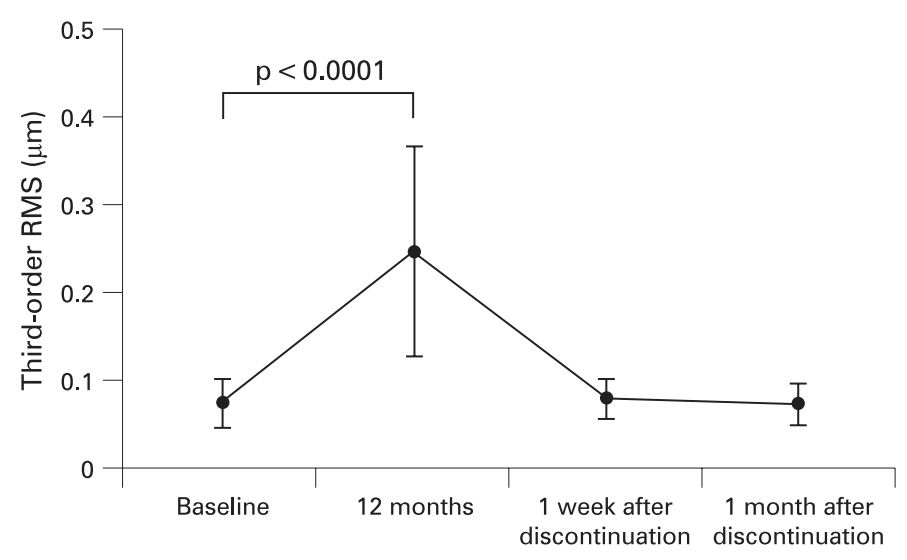

Time course

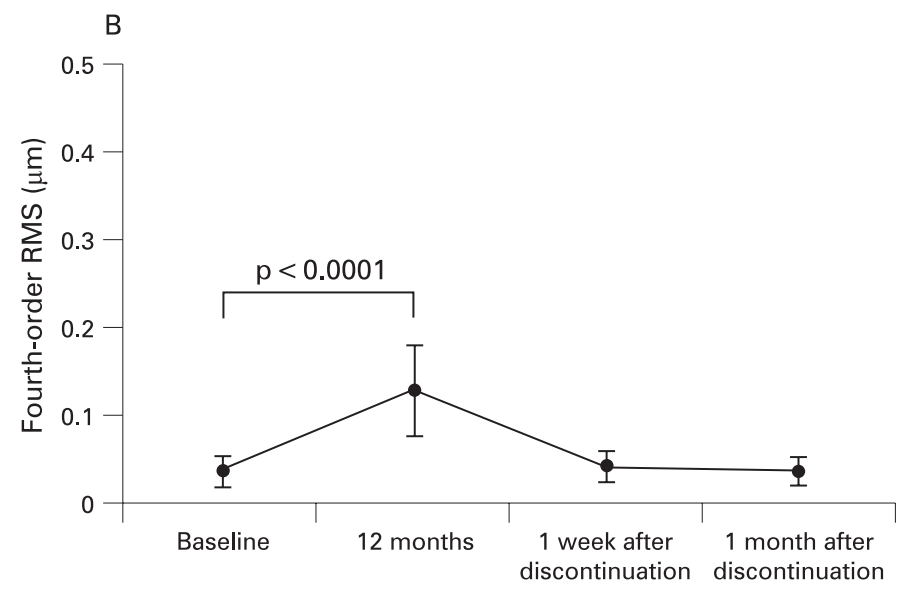

Time course

Figure 4 Time course of changes in ocular higher-order aberrations for a $4 \mathrm{~mm}$ pupil after commencement and discontinuation of overnight orthokeratology. RMS, root mean square. (A) There were significant increases in third-order RMS for a 4 mm pupil at 12 months after commencement of the procedure $(p<0.0001$, Dunnett test). One week after discontinuation of the procedure, the complete recovery without significant difference from the baseline value $(p=0.9932)$ was observed. One month after the discontinuation, it maintained the recovered level with no significant difference from the pretreatment value $(p=0.9999)$. Error bars represent the SD. (B) Significant increases in fourth-order RMS for a $4 \mathrm{~mm}$ pupil were found at 12 months after commencement of the procedure $(p<0.0001$, Dunnett test). After discontinuation of the procedure, it returned to the baseline level; there was no significant difference between values at baseline and at 1 week $(p=0.9337)$, and between those at baseline and at 1 month after the cessation. $(p=0.9999)$. Error bars represent the SD.

discontinuation of the procedure in children and young people. Many practitioners are concerned about the influence of this procedure on the development of the eye. A similar study for children and young people is certainly needed to elucidate this point.

In conclusion, we have for the first time confirmed the complete reversibility of overnight orthokeratology procedure in light of optical quality of the eye and quality of vision as well as refraction and visual acuity. On the basis of these findings, we can explain to patients that overnight orthokeratology is a reversible procedure. In addition, the current information concerning each recovery time is quite useful to consider the

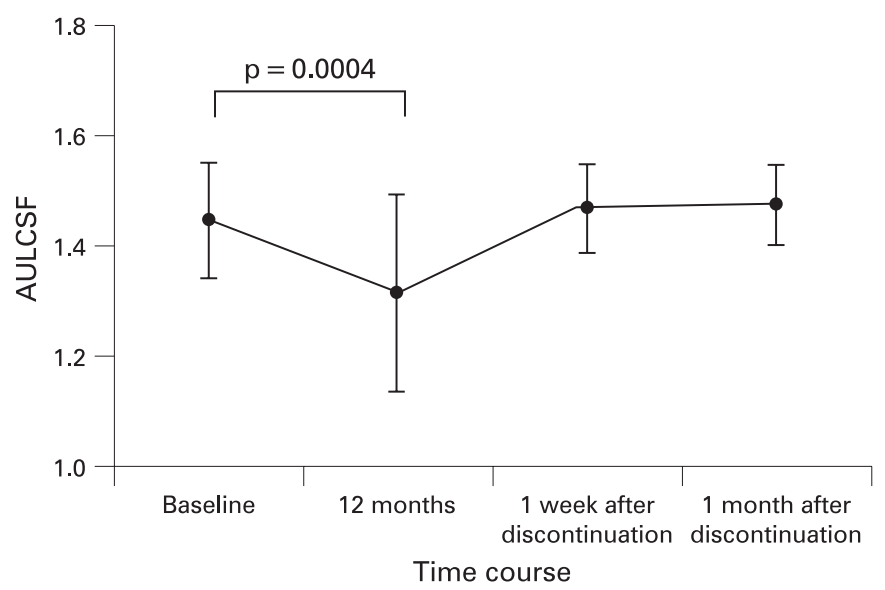

Figure 5 Time course of changes in area under the log contrast sensitivity function (AULCSF) after commencement and discontinuation of overnight orthokeratology. AULCSF decreased significantly 12 months after commencement of the procedure ( $p=0.0004$, Dunnett test). At 1 week after discontinuation of the procedure, it recovered completely to the baseline level without any significant difference from the baseline value $(p=0.9962)$, and thereafter remained at the recovered level until 1 month with no significant difference from the pretreatment value $(p=0.9999)$. Error bars represent the SD. starting-point of the following procedure, if patients undergoing orthokeratology hope for another corrective option, such as laser refractive surgery.

Funding: None.

Competing interests: None.

Ethics approval: Ethics approval was provided by the institutional review board of Tsukuba University Hospital.

Patient consent: Patient consent was obtained.

\section{REFERENCES}

1. Nichols JJ, Marsich MM, Nguyen M, et al. Overnight orthokeratology. Optom Vis Sci 2000; 77:252-9.

2. Rah MJ, Jackson JM, Jones LA, et al. Overnight orthokeratology: preliminary results of the Lenses and Overnight Orthokeratology (LOOK) study. Optom Vis Sci 2002;79:598-605.

3. Cheung SW, Cho P. Subjective and objective assessments of the effect of orthokeratology - a cross-sectional study. Curr Eye Res 2004;28:121-7.

4. Swarbrick HA. Orthokeratology review and update. Clin Exp Optom 2006;89:12443.

5. Haque S, Fonn D, Simpson T, et al. Corneal and epithelial thickness changes after 4 weeks of overnight corneal refractive therapy lens wear, measured with optical coherence tomography. Eye Contact Lens 2004;30:189-93.

6. Barr JT, Rah MJ, Meyers W, et al. Recovery of refractive error after corneal refractive therapy. Eye Contact Lens 2004;30:247-51.

7. Soni PS, Nguyen TT, Bonanno JA. Overnight orthokeratology: refractive and corneal recovery after discontinuation of reverse-geometry lenses. Eye Contact Lens 2004;30:254-62.

8. Sorbara L, Fonn D, Simpson T, et al. Reduction of myopia from corneal refractive therapy. Optom Vis Sci 2005;82:512-18.

9. Rengstorff RH. Corneal curvature and astigmatic changes subsequent to contact lens wear. J Am Optom Assoc 1965;36:996-1000.

10. Rubin ML. The tale of the warped cornea: a real-life melodrama. Arch Ophthalmol 1967;77:711-12.

11. Levenson DS, Berry CV. Findings on follow-up of corneal warpage patients. CLAO J 1983;9:126-9.

12. Wilson SE, Lin DT, Klyce SD, et al. Topographic changes in contact lens-induced corneal warpage. Ophthalmology 1990;97:734-44.

13. Ruiz-Montenegro J, Mafra CH, Wilson SE, et al. Corneal topographic alterations in normal contact lens wearers. Ophthalmology 1993;100:128-34.

14. Hartstein J. Keratoconus that developed in patients wearing corneal contact lenses. Report of four cases. Arch Ophthalmol 1968;80:345-6.

15. Nauheim JS, Perry HD. A clinicopathologic study of contact-lens-related keratoconus. Am J Ophthalmol 1985;100:543-6.

16. Macsai MS, Varley GA, Krachmer JH. Development of keratoconus after contact lens wear. Patient characteristics. Arch Ophthalmol 1990;108:534-8. 
17. Hiraoka T, Furuya A, Matsumoto $Y$, et al. Quantitative evaluation of regular and irregular corneal astigmatism in patients having overnight orthokeratology. J Cataract Refract Surg 2004;30:1425-9.

18. Hiraoka T, Matsumoto $Y$, Okamoto F, et al. Corneal higher-order aberrations induced by overnight orthokeratology. Am J Ophthalmol 2005;139:429-36.

19. Joslin CE, Wu SM, McMahon TT, et al. Higher-order wavefront aberrations in corneal refractive therapy. Optom Vis Sci 2003;80:805-11.

20. Hiraoka T, Okamoto C, Ishii $Y$, et al. Contrast sensitivity function and ocular higherorder aberrations following overnight orthokeratology. Invest Ophthalmol Vis Sci 2007:48:550-6.

21. Berntsen DA, Barr JT, Mitchell GL. The effect of overnight contact lens corneal reshaping on higher-order aberrations and best-corrected visual acuity. Optom Vis Sci 2005;82:490-7.
22. Cheung SW, Cho P, Chui WS, et al. Refractive error and visual acuity changes in orthokeratology patients. Optom Vis Sci 2007;84:410-16.

23. Stillitano I, Schor $\mathrm{P}$, Lipener $\mathrm{C}$, et al. Stability of wavefront aberrations during the daytime after 6 months of overnight orthokeratology corneal reshaping. J Refract Surg 2007;23:978-83.

24. Oshika T, Tomidokoro A, Maruo K, et al. Quantitative evaluation of irregular astigmatism by Fourier series harmonic analysis of videokeratography data. Invest Ophthalmol Vis Sci 1998;39:705-9.

25. Kuroda T, Fujikado T, Maeda N, et al. Wavefront analysis of higher-order aberrations in patients with cataract. J Cataract Refract Surg 2002;28:438-44.

26. Applegate RA, Howland HC, Sharp RP, et al. Corneal aberrations and visual performance after radial keratotomy. J Refract Surg 1998;14:397-407.

27. Swarbrick HA, Wong G, O'Leary DJ. Corneal response to orthokeratology. Optom Vis Sci 1998; 75:791-9. 